\title{
Incidencia de los Programas de Formación Pedagógica en el Perfil de los Profesionales No Licenciados en la Facultad de Educación de UNIMINUTO
}

\author{
María V. Rodríguez-Perez ${ }^{(1)^{*}}$ y Francisco J. Hinojo-Lucena ${ }^{(2)}$ \\ (1) Corporación Universitaria Minuto de Dios: Carrera 74 81C-05, Bogotá, D. C., Colombia. \\ (e-mail: maria.rodriguezp@uniminuto.edu,vickyr.rodriguez@gmail.com) \\ (2) Universidad de Granada: Av. del Hospicio, s/n, 18010 Granada, España. (e-mail: fhinojo@ugr.es) \\ ${ }^{*}$ Autor a quien debe ser enviada la correspondencia
}

Recibido Ene. 26, 2017; Aceptado Mar. 29, 2017; Versión final May. 2, 2017, Publicado Oct. 2017

\begin{abstract}
Resumen
El objetivo del artículo es determinar la incidencia de la formación en pedagogía en el perfil del educador no licenciado en el Programa de Pedagogía para Profesionales No Licenciados de la Facultad de Educación de UNIMINUTO, en la ciudad de Bogotá, Colombia. Se utilizó un diseño metodológico mixto, transversal, de alcance descriptivo. A la información recolectada usando como instrumento un cuestionario, se aplicó la escala Likert en una muestra de 201 estudiantes. Los resultados arrojan porcentajes similares entre los estudiantes que están de acuerdo y los que están en desacuerdo en que el programa incide favorablemente en la cualificación del perfil del educador no licenciado. En conclusión, se evidencia la necesidad de implementar acciones de mejora como la ampliación del tiempo para el desarrollo del seminario de pedagogía, la reestructuración de la evaluación y la posible homologación de créditos del programa para estudios de postgrado.
\end{abstract}

Palabras clave: formación en pedagogía; programas de pedagogía; funciones de docencia; profesional no licenciado; calidad de la educación

\section{Incidence of the Teacher Training Programs in the Profile of the Not-Graduate Professionals in the Faculty of Education of UNIMINUTO}

\begin{abstract}
The objective of the article is to determine the incidence of training in pedagogy in the profile of the notgraduate educator in the educational program for not-graduate professionals of the Faculty of Education of UNIMINUTO, in the city of Bogota, Colombia. A mixed methodological design, transversal and scope descriptive was employed. The information collected using a questionnaire as the main instrument was subjected to analysis applying a Likert scale in a sample of 201 students. The results show similar percentages between students who are in agreement and those who are in disagreement that the program has a favorable effect on the qualification of the profile of the not-graduate educator. In conclusion, it is observed that it is necessary to implement actions for improvement such as increasing the time for the development of the Seminar of Pedagogy, the restructuration of the evaluation and the possible homologation of credits for the graduate studies program.
\end{abstract}

Keywords: training in pedagogy; teaching programs; functions of teaching; not licensed professional; quality of education 


\section{INTRODUCCIÓN}

Con la llegada de la globalización y la modernidad a las sociedades emergen desafíos en cuanto a la calidad de la educación en todos los países del mundo. Esta situación le ha dado al tema una posición privilegiada en los diálogos intersectoriales entre quienes inciden directamente sobre el destino de la educación. En este orden, se anuncia la necesidad de contar con educadores que estén en capacidad de transformar los sistemas educativos y así contribuir al mejoramiento de la educación. En el caso particular de Colombia, en las dos últimas décadas, la calidad de la educación ha sido uno de los asuntos más discutidos y a la vez más complejos. En esta línea, el Ministerio de Educación Nacional -MEN, en el Plan Sectorial de Educación 20102014, planteó que "(...) una educación de calidad y pertinente para el siglo XXI, permite que las personas estén preparadas para obtener, adaptar y aplicar la información en múltiples contextos y redes y transformarla en un conocimiento relevante para la satisfacción de sus necesidades" (MEN, 2010).

En la comprensión de cuáles serían los factores asociados a la calidad de la educación colombiana se resalta la formación de los educadores. Es así como en el señalamiento político de los objetivos y las líneas de acción, el MEN plantea que una educación de calidad depende esencialmente del compromiso y del apoyo de los docentes y, por lo mismo, se deben promover procesos para su mejoramiento profesional a través de programas de excelencia en su formación. En este marco, en el Plan Decenal de Educación 2006-2016, se ha planteado que, para avanzar en la calidad de la educación, se requiere de docentes con una sólida formación, "(...) cuyos ejes esenciales sean la pedagogía, la ciencia, el arte, la tecnología, la investigación, la ética y los derechos humanos" (MEN, 2006).

Al respecto, Imbernón (1989) plantea que, para optimizar la calidad de la educación, se debe garantizar antes una excelente formación docente, tarea que solo se podría alcanzar si las instituciones de educación superior (IES) se comprometen con la calidad de la educación del país a través de programas de formación docente. Clark (1995), en el texto Pasión por enseñar, hablando del rol del docente afirma que "(...) todas las influencias sobre la calidad de la educación están mediadas por él y por su acción. Tienen la posibilidad de aumentar la calidad de la educación (...). Y también pueden degradar la calidad de la educación” (Day, 2006, p. 15). En este sentido, es evidente que la labor de los educadores cumple un papel fundamental en la calidad de los procesos de enseñanza y aprendizaje, razón que invita a reflexionar sobre la actual formación docente. Estamos en un siglo de constante transformación; por lo mismo, estos programas están convocados a la actualización permanente de los currículos que constituyen las ofertas académicas para el profesorado. Asimismo, Fullan (1998), en el artículo titulado "Iniciativas mundiales para mejorar la formación de profesores", indica que, además de atraer personas capaces y con gran sentido de compromiso, para generar un cambio importante en la formación docente de debe incrementar su base de conocimiento (citado en Vaillant, 2010). Al respecto, el MEN ha estado insinuando la impostergable necesidad de que los mejores bachilleres lleguen a las facultades de educación, situación que no es fácil por las mismas condiciones en que se ha ido desarrollando la formación de los docentes en Colombia.

En el contexto de las experiencias internacionales exitosas en el logro de la calidad de la educación, los sistemas educativos de Singapur, Finlandia, Canadá (Ontario) y Corea del Sur son un marco de referencia que da cuenta del más alto desempeño. Según García et al. (2014), estos países, actualmente reconocidos como "naciones del conocimiento", han cimentado las propuestas de políticas de calidad de la educación en la formación de los docentes, teniendo como foco aspectos relacionados con "i) formación previa al servicio, ii) selección, iii) retención y promoción, iv) evaluación para el mejoramiento continuo, v) formación en servicio y vi) remuneración" (p. 21). Los autores, en las conclusiones de su estudio, señalan que en estos países “(...) hay pocos y muy selectivos programas de formación docente a nivel universitario, de muy alta calidad, que enfatizan la práctica y la investigación pedagógica" (p. 21). Esta investigación deja en evidencia que la centralización y la calidad de los programas de formación docente, que garantizan los Gobiernos en los países referenciados, no han permitido la oferta descontrolada de los mismos, entre otras cosas, porque la selección de los docentes se realiza mediante un largo y exigente proceso. Este modelo de reclutamiento de docentes debería ser considerado en Colombia para responder a las expectativas que plantea el Gobierno en la visión del Plan Nacional de Desarrollo 2014-2018: "Colombia será el país más educado de América Latina en 2025, con un capital humano capaz de responder a las necesidades locales y globales" (p. 83).

La heterogeneidad y la diversificación son las características dominantes de la formación de los docentes en América Latina; no importando su nivel, se forman en instituciones de todo tipo: en escuelas normales, en institutos de enseñanza superior, en institutos provinciales o municipales, en instituciones superiores de enseñanza técnica, en universidades, en instituciones privadas y, además, están los que se desempeñan como docentes sin tener un título que los habilite para tal labor (Vaillant, 2006). Esta proliferación de instituciones y programas de formación docente impacta desfavorablemente en el futuro de la profesión. 
En Colombia hay una oferta excesiva de programas de formación docente, con el agravante de que pocos tienen reconocimiento en acreditación de alta calidad. En este contexto, Ariza (2014) plantea que "la excelencia docente implica enfrentar los temas desde la raíz; (...) deberán ser nuestras licenciaturas las profesiones más destacadas de la educación superior" (p. 56). El reto de replantear los programas de formación docente ha promovido la formulación de nuevas políticas educativas que se ocupan en exclusividad de las licenciaturas en educación, dejando pasar inadvertida la existencia de otros programas conducentes a otorgar títulos que acreditan idoneidad para desarrollar funciones de docencia, como es el caso de los Programas de Pedagogía para Profesionales No Licenciados (PPPNL).

García et al. (2014), como producto de la investigación Tras la excelencia docente: Cómo mejorar la calidad de la educación para todos los colombianos, proponiendo unos ejes de transformación, sugieren que una condición para alcanzar esta meta es "(...) reglamentar y certificar los programas de pedagogía que deben cursar los profesionales no licenciados, con miras a que, en el mediano plazo, todos los programas de nivelación se conviertan en maestrías que sigan parámetros de alta calidad" (p. 31); en el mismo sentido, indican que existe la necesidad de que"(...) los requisitos de entrada de futuros docentes al magisterio sean más rigurosos, para que en un lapso de diez años se asegure que ingresen sólo los mejor formados -con mínimo cuatro años de formación universitaria- y quienes demuestren la mejor vocación y carácter para ejercer la docencia" (p. 31). Estos señalamientos son una crítica al proceso actual de vinculación de docentes al sistema educativo colombiano, puesto que para su ingreso no se tiene contemplado una formación en educación previa al servicio, y los programas de PPPNL escasamente cumplen con las 480 horas reglamentadas, siendo la causa por la cual los profesionales no licenciados adquieren un limitado conocimiento de la pedagogía.

Vezub (2007) afirma que en muchos casos no se parte del diagnóstico de las futuras necesidades profesionales ni se analizan cuáles son las áreas por desarrollar ante las demandas y problemáticas de la docencia hoy en día, antes de ofrecer propuestas de capacitación que normalmente obedecen más bien a sus propios intereses y posibilidades. "Orientar la oferta de formación continua en relación con las necesidades y problemas detectados en los sistemas educativos parece una exigencia básica, pero aun irrealizada" (p. 17).

En esta perspectiva, Predaja et al. (2012) en el artículo "Sistema de formación pedagógica en la universidad de Otavalo en Ecuador", afirman que en la formación del recurso humano avanzado, y más específicamente en la formación docente, el rol de las universidades en las nuevas sociedades del conocimiento ha adquirido gran importancia (citados por Acosta, Abreu y Coronel, 2015). En este mismo sentido, Marcelo y Vaillant (2009) plantean que "en la formación inicial (...) tenemos la oportunidad de crear nuevos docentes apasionados por la enseñanza (...). Para que este objetivo se cumpla (...) debemos plantear la necesidad imperiosa de reformar las estructuras curriculares, organizativas y personales que actualmente imperan" ( $p$. 49). En conjunto, estos diversos señalamientos dejan en evidencia que la calidad de los programas de formación y cualificación docente deben centrarse en el saber esencial de la pedagogía, ciencia que al ser estudiada promueve el conocimiento de otras teorías aliadas a la educación.

La vinculación de profesionales no licenciados mediante el Decreto 1278 (2002) ha promovido, en los expertos en el tema de la calidad de la educación y la formación docente, el interés por indagar sobre las implicaciones que tiene esta normatividad, tanto en la carrera docente como en los profesionales no licenciados que, sin contar con formación previa en pedagogía, desarrollan labores de docencia. Al respecto, Cuervo (2013), en su trabajo "La inserción de profesionales docentes no licenciados al sistema público educativo: ¿Qué hay en su quehacer pedagógico?", plantea como objetivo identificar y analizar la dinámica de los profesionales no licenciados en su práctica docente. Algunos de los resultados, en cuanto al nivel de formación de ingreso, muestran que los docentes no licenciados se vinculan con título profesional, en principio, y solo algunos de ellos con estudios de especialización; no obstante, aun cuando los PNL ostenten un dominio avanzado de las competencias básicas, en ámbitos prácticos sus posibilidades de mediación didáctica son insignificantes. Esta investigación permite percibir que tanto el saber disciplinar como la pedagogía y la didáctica son complementos indispensables en la acción educativa que desarrolla un docente.

En la investigación de Castro y Barrantes (2011), Hacia una caracterización de los profesionales no licenciados en educación (PNL): Percepciones y realidades, los hallazgos develan que "(...) el programa de formación pedagógica exigido a los PNL debería ser una condición para participar en el concurso. De esta manera se podría seleccionar personas que aspiran a la docencia. (...) El componente pedagógico debe ser objeto de evaluación, pues la mayoría de profesionales reconocen que pasaron el concurso porque en las diferentes pruebas se privilegió el aspecto disciplinar" (p. 84). Esta investigación realiza una aproximación más cercana a la realidad en que se ha estado desarrollando la labor docente de los PNL. Asumir funciones pedagógicas sin tener formación en el campo de la educación ha traído consecuencias poco favorables tanto para los educadores no licenciados como para los que sí lo son, lo que da lugar a que esta profesión no se proyecte claramente en concordancia con la calidad de la educación que se busca en el país. 
Durán, Acosta y Espinel (2014) en su artículo "Experiencia docente de profesionales no licenciados en la escuela pública del Distrito", publican los resultados de la caracterización de la experiencia docente de quienes, a partir del Decreto 1278, pudieron vincularse en las escuelas públicas del Distrito Capital. Los resultados señalan que "con la incursión de sujetos sin formación pedagógica en la enseñanza escolar se evidencia la realidad del concepto de desprofesionalización, dado que tanto la pedagogía como la didáctica pierden su sentido en el ejercicio de la enseñanza" (p. 56). Otra de las conclusiones del equipo investigador tiene que ver con que los propósitos del Gobierno en la Ley General de Educación (1994) sobre la mejora de la calidad de la educación, resumidos en el Decreto 1278, se ven mermados al no asegurar la implementación de programas de educación pedagógica y didáctica a los PNL que se incorporan, con el fin de garantizar que se optimice la calidad de los conocimientos de orden científico impartidos.

Este antecedente deja en evidencia la relevancia que tiene la pedagogía y la didáctica en la formación de los educadores y, por efecto, en la calidad de la educación. El panorama anterior persuade a las instituciones de educación superior con PPPNL a no continuar "dando de lo mismo"; por el contrario, deben asumir la tarea de revisar estos programas con el fin de reorientarlos hacia nuevas perspectivas que permitan al educador sin formación en pedagogía asumir las funciones de docencia con la altura con que las desarrolla un licenciado en educación.

\section{PROGRAMA DE PEDAGOGÍA PARA PROFESIONALES NO LICENCIADOS}

La Corporación Universitaria Minuto de Dios -UNIMINUTO es una institución de educación superior colombiana, de carácter privado. La Escuela de Alta Docencia -EAD es una unidad académica de la Facultad de Educación creada para el perfeccionamiento constante del conocimiento y la práctica de la enseñanza inherente a la educación, que se ocupa de la formación del sujeto en los diferentes aspectos, aristas y significados de su dimensión histórica, humana y social (UNIMINUTO, 2011).

El Programa de Pedagogía para Profesionales No Licenciados (PPPNL) es una de las ofertas académicas de la EAD desde hace más de diez años. Este programa, que es el objeto de análisis de la presente investigación, cuenta con quince docentes. Los estudiantes del PPPNL son profesionales no licenciados que buscan en el programa formación en pedagogía y didáctica, con el fin de tener un mejor desempeño en el ejercicio docente y, por ende, una cualificación en sus perfiles como educadores. Estos profesionales se encuentran laborando en el sector educativo público en virtud de una reglamentación diferencial establecida en el Decreto 1278 de 2002, en la que se plantea que "(...) los profesionales con título diferente al de licenciado en educación, deben acreditar, al término del periodo de prueba, que cursan o han terminado un postgrado en educación, o que han realizado un programa de pedagogía, de acuerdo con la reglamentación que al respecto expida el Gobierno Nacional" (Art. 12 $2^{\circ}$, par. 1). De lo anterior se colige que los profesionales vinculados al sistema educativo que no tengan titulación que acredite su idoneidad para desarrollar funciones de docencia, deben asumir simultáneamente su formación en pedagogía a la vez que ejerzan la labor pedagógica.

En tal sentido, UNIMINUTO, en su compromiso con la formación de educadores y la calidad de la educación del país, se propuso desarrollar un programa para los educadores no licenciados bajo los lineamientos iniciales de la normatividad. En un comienzo, esta propuesta, que se denominó Programa de Ingreso al Escalafón, exigía como mínimo 360 horas de estudio, distribuidas en un año de trabajo. Fue así como, a través de este programa, orientado en principio a fortalecer la formación en Tecnología e Informática, se entregaron trece promociones. Luego se realizaron modificaciones en denominación, número de créditos y estructura curricular en concordancia con las exigencias del Decreto 2035 de 2005, que establece los objetivos y los requisitos del programa de pedagogía que deben acreditar los profesionales con título diferente al de licenciados en educación al término del periodo de prueba; el decreto también se señala que las instituciones de educación superior que ofrezcan el programa deberán estructurar un conjunto de acciones formativas en las que se destaque la pedagogía como saber fundante de la educación (Artículo 4º); en consecuencia, la pedagogía se toma como componente fundamental en la formación de los estudiantes en los PPPNL.

En la actualidad, la EAD, para el desarrollo del PPPNL, tiene planteada una intensidad de 480 horas representadas en 10 créditos académicos. La estructura curricular está conformada por doce seminarios que se desarrollarán mediante encuentros sincrónicos los días sábados y un componente amplio de trabajo asincrónico. Con el seminario de Conceptualización y Fundamentos de la Pedagogía, que está considerado como el pilar del proceso de formación del programa, se busca profundizar en los saberes de esta disciplina y las teorías afines a la educación; está organizado para ser desarrollado en 16 horas presenciales (ocho horas diarias, dos sábados), más 80 horas de formación autónoma, que en su totalidad equivalen a 96, es decir, dos créditos académicos. En comparación con un programa de Licenciatura en Educación que cuenta con 155 créditos académicos en los que la práctica pedagógica y el saber pedagógico ocupan un lugar privilegiado, se puede percibir que el tiempo de ejecución del programa y, más aun, de los seminarios de pedagogía y didáctica es todavía limitado para la cualificación del perfil del educador no licenciado. 
En este contexto, el objetivo de esta investigación es estudiar la incidencia del PPPNL en la cualificación del perfil del educador no licenciado a partir de la opinión que han ido construyendo los estudiantes del programa a través de su percepción, para detectar posibles áreas de mejora que favorezcan la formación en pedagogía de quienes ejercen la labor educativa sin contar con conocimientos previos en educación. Proponer un plan de acciones que fortalezca el programa coadyuvará, sin lugar a dudas, a la calidad de la formación de docentes y, con ello, a la calidad de la educación colombiana.

\section{LA PEDAGOGÍA EN LA FORMACIÓN DOCENTE}

El reconocimiento de la formación pedagógica del educador ha sido uno de los aspectos más discutidos al plantear la reestructuración de los programas de formación docente. Vaillant y Marcelo (2015) consideran que "la dimensión cognitivo-académica en la que se incluyen la cualidad y cantidad de saberes del docente (...), sus estrategias pedagógicas y su efectividad en la trasmisión y construcción de conocimiento" (p. 12) son elementos esenciales en su formación. En otro estudio (2009), estos mismos autores insisten en que es ineludible que el maestro como parte esencial de su oficio, además de las competencias pedagógicas, deba dominar con suficiencia la asignatura que enseña.

La pedagogía como ciencia de la educación "(...) es un espacio de producción de capital simbólico -saberes pedagógicos, teóricos y prácticos-sobre la educación, y de capital social en tanto formación de profesionales" (Juliao, 2014, p. 23). En el plano de la formación docente, la pedagogía es uno de los grandes desafíos de las facultades de educación. Al respecto, Acosta et al., indican que si se quiere generar una transformación, a conciencia, hacia una desarrollo pedagógico óptimo, los futuros docentes deben adquirir la capacidad del autoanálisis, para efecto de los cual deben recibir de las universidades las herramientas y las estrategias pertinentes.

Aguerrondo (2009) afirma que “(...) una primera dimensión de la competencia profesional docentes es, sin duda, el manejo adecuado de la pedagogía (...) como base científica de su accionar (p. 368). En esta dirección, Juliao (2014) afirma que "hay pedagogía cuando se reflexiona sobre la educación, cuando el saber educar, implícito e intuitivo en toda sociedad (...) se convierte en un saber sobre y desde la educación" (p. 22). Además, plantea que "el desarrollo de la pedagogía implica la sistematización de dicho saber, de sus modelos, métodos y procedimientos, y la delimitación de su objetivo concreto" (p. 23). Consecuente con lo anterior, la pedagogía es la configuración de la teoría y la práctica que caracterizan la labor docente y con ella la permanente reflexión de su acción educativa. Vaillant y Marcelo (2015) señalan que, para enseñar, el docente, además de su conocimiento específico, deberá contar con competencias y conocimientos pedagógicos generales; esto es, sobre los principios universales del proceso enseñanza-aprendizaje, los estudiantes, los tiempos, las técnicas didácticas, la estructura de las clases y, más generalmente, sobre la planificación de la enseñanza, las teorías del desarrollo humano, la planificación curricular, la evaluación; por supuesto, sin dejar de lado cuestiones más globales como la filosofía e historia de la educación y la influencia de los contextos socioculturales en la enseñanza.

La formación pedagógica del profesorado, en el marco de la educación superior y las políticas educativas, representa una tarea de primer orden, de necesidad impostergable y de elevada complejidad. En esta línea, Rentería (2004) plantea que "la formación intelectual del maestro necesariamente deberá estar determinada por el conocimiento de la pedagogía, tanto en su campo practico como teórico" (p. 66); es decir que, para desarrollar las características de un intelectual universal, el maestro, como base de su formación, tendrá que adquirir el dominio de temas prioritarios en el área de la educación y la pedagogía.

Como se ha ido señalando, el conocimiento de la pedagogía en la formación docente lleva a convertirla en el objeto principal del saber del educador; de ahí la responsabilidad de las IES en la calidad de los programas que ofertan en las facultades de educación. En este contexto, Giroux (2016) expresa que "como educadores necesitamos reconocer que las formas más importantes de dominación no son solo económicas sino también intelectuales y pedagógicas" (p. 19). En otro estudio, el mismo autor (1997) insiste en que "(...) urge desarrollar programas que posibiliten que los futuros profesores sean educados como intelectuales transformativos" (1997, p. 210). Fullan (1998) indica que para cualificar la formación docente sería necesario generar una base de conocimiento mayor para la docencia y atraer estudiantes capaces, diversos y comprometidos con la profesión (citado por Vaillant, 2010).

Marcelo y Vaillant (2009) argumentan que existe una clara descoordinación y fragmentación entre los diferentes tipos de conocimiento en las mallas curriculares de los programas de formación docente, pues no hay una conexión directa entre los contenidos disciplinares y los pedagógicos; citando a Feiman-Nemser, hacen énfasis en que entre la formación inicial y la posterior realidad escolar hay una evidente escisión, pues se percibe que lo que se imparte en la universidad, poco tiene que ver con las competencias y los conocimientos que se requieren en las práctica pedagógicas. 
"Es así como la formación pedagógica no puede ser solo vista como un requisito para los educadores (...); hay tanta disparidad y estrategias metodológicas como instituciones que la desarrollan. En los programas se encuentran diferencias en concepciones, tiempos de duración -de un año a cuatro meses-, diferentes estrategias para su desarrollo - presenciales, virtuales, semipresenciales-, disparidad de intencionalidades y contenidos. Esta realidad en la formación pedagógica afecta la profesión docente y, lo peor, la calidad de los procesos educativos que se ofrecen a los estudiantes" (Castro y Barrantes, 2011, p.84). Lo señalado hasta aquí deja en evidencia la influencia de la política educativa en los sistemas educativos y, a la vez, muestra, desde diversas perspectivas, que los educadores son los protagonistas de la calidad de la educación; por tanto, son las universidades quienes deben garantizar que los programas de formación previa al servicio y cualificación docente sean de calidad.

\section{METODOLOGÍA}

La población, objeto de estudio, correspondió a 350 estudiantes de las cohortes 2015 y 2016 del PPPNL, de una universidad privada colombiana: la Corporación Universitaria Minuto de Dios -UNIMINUTO. La muestra obtenida fue de 201 sujetos que culminaron sus estudios, de los cuales 120 son hombres (60\%) y 81 mujeres (40\%), con edades entre los 30 y 55 años, elegidos por muestreo no probabilístico por juicio, con base en criterios que permitieron al investigador asegurar el acceso a la muestra más representativa. "Aquí, el procedimiento no es mecánico ni con base en fórmulas de probabilidad, sino que depende del proceso de tomas de decisiones de un investigador (...) y, desde luego, las muestras seleccionadas obedecen a otros criterios de investigación" (Hernández, et al., 2010).

Esta es una metodología de alcance descriptivo, con un diseño transversal, en la medida en que no se busca identificar cambios a lo largo del tiempo sino determinar el grado de incidencia en un periodo específico; la obtención de datos se realizó a través de la aplicación de un cuestionario aplicado a 201 estudiantes que culminaban el PPPNL. Se utilizó una escala Likert, compuesta de 31 ítems agrupados en tres dimensiones: 1) motivación intrínseca; 2) motivación extrínseca, y 3) satisfacción percibida sobre el aporte del PPPNL en su formación y cualificación del perfil docente, donde 4 es totalmente de acuerdo; 3, de acuerdo; 2, poco acuerdo, y 1 , totalmente en desacuerdo. Del instrumento, se obtuvo una confiabilidad de Alfa de Cronbach de 0,952 , lo cual indica una adecuada consistencia interna de las preguntas. Los ítems de la escala Likert fueron enviados en un formulario por correo electrónico y el tiempo de respuesta por parte de los alumnos fue de 15 días. La información fue analizada a través del programa SPSS v. 22,0. Se llevó a cabo un análisis de factores de las tres dimensiones y la extracción se realizó por componentes principales (ver Tabla 1).

\section{RESULTADOS Y DISCUSIÓN}

Los resultados se presentan en tres secciones, en las que se analizan tres factores: 1) motivación intrínseca; 2) motivación extrínseca, y 3) percepción sobre el aporte del PPPNL en la formación y cualificación del perfil docente del educador no licenciado.

\section{Factor 1. Motivación intrínseca}

Con relación a la variable de motivación intrínseca, se preguntó a los estudiantes las razones por las que eligieron cursar el PPPNL ofertado en UNIMINUTO; se halló que el $93 \%$ coinciden en que una de las motivaciones para cursar el programa en estudio tiene que ver con la adquisición de conocimientos en pedagogía. La función de la pedagogía en la formación de los educadores no licenciados es una necesidad de primer orden para su labor educativa. La profesión docente en la última década ha llamado la atención de diversos profesionales para vincularse al sistema educativo, en la investigación se encontró que el $85 \%$ manifestaron estar de acuerdo con que cursaron el programa por iniciativa propia, porque estaban interesados en ejercer labores de docencia. Los programas de formación docente permiten de cualquier modo transformar la concepción inicial que se trae sobre educación, en este sentido, se indagó si la formación en pedagogía les había permitido modificar la concepción inicial que tenían sobre educación; el 70 \% respondió estar de acuerdo; además, el 82 \% de los estudiantes percibió la profesión docente como una mejor oportunidad laboral que la que representa la profesión en la que se han formado. Para finalizar, el $63 \%$ de los estudiantes afirma que los resultados de los procesos formativos en el programa cumplieron con las expectativas que tenían, sin embargo, se evidencia que el porcentaje restante de los encuestados no están de acuerdo, razón que lleva a revisar los factores que incidieron para que se diera esta respuesta (ver Tabla 1). 
Tabla 1: Análisis factorial de las variables que estudian las motivaciones y la satisfacción sobre el Programa de Pedagogía para Profesionales No Licenciados (PPPNL).

\begin{tabular}{|c|c|c|}
\hline \multirow{2}{*}{$\begin{array}{l}\text { Variables relacionadas con } \\
\text { la motivación intrínseca }\end{array}$} & Contenido de la variable & \multirow{2}{*}{$\begin{array}{l}\text { Coefi- } \\
\text { cientes }\end{array}$} \\
\hline & $\begin{array}{l}\text { Las razones por las que creo que he elegido cursar el Programa de } \\
\text { Pedagogía para profesionales no Licenciados en UNIMINUTO }\end{array}$ & \\
\hline 8.2 Saber pedagógico & Adquirir conocimientos en Pedagogía & 0.712 \\
\hline 8.5 Iniciativa propia & Iniciativa propia porque estoy interesado (a) en ejercer labores en docencia & 0.696 \\
\hline $\begin{array}{l}9.12 \text { cambios en la } \\
\text { concepción inicial sobre } \\
\text { educación }\end{array}$ & $\begin{array}{l}\text { La formación en Pedagogía me ha permitido modificar la concepción de } \\
\text { Educación que tenía. }\end{array}$ & 0.641 \\
\hline $\begin{array}{l}8.6 \text { mejor oportunidad } \\
\text { laboral }\end{array}$ & $\begin{array}{l}\text { Porque veo en la profesión docente una mejor oportunidad laboral que en la } \\
\text { que me he formado }\end{array}$ & 0.664 \\
\hline 9.20 Referir el programa & $\begin{array}{l}\text { A partir de mi experiencia como estudiante del Programa lo recomendaría a } \\
\text { una persona }\end{array}$ & 0.592 \\
\hline $\begin{array}{l}\text { 9.31 Satisfacción con el } \\
\text { programa }\end{array}$ & $\begin{array}{l}\text { Los resultados de la formación en Pedagogía para los profesionales no } \\
\text { licenciados, cumplió con las expectativas que los estudiantes esperaban. }\end{array}$ & 0.587 \\
\hline
\end{tabular}

\section{Factor 2. Motivación extrínseca}

Pedagogía y didáctica en la formación docente son saberes sobre los cuales se representa el oficio docente. En el estudio se preguntó a los participantes sobre las motivaciones extrínsecas; se indagó su percepción sobre si el PPPNL lo cursaban para mejorar las prácticas de enseñanza; el $86 \%$ reconocieron que el programa contribuye a optimizar las didácticas que aplican en los procesos de enseñanza; el $78 \%$ dijo estar de acuerdo con que una de las razones por las que creen que han elegido cursarlo es para mejorar sus ingresos económicos. Los educadores no licenciados vinculados mediante Decreto Ley 1278, de no realizar un curso de formación en pedagogía al término del periodo de prueba son desvinculados del sistema público educativo, razón por la cual el $93 \%$ de los indagados estuvo de acuerdo con que lo hacían porque era un requisito para ejercer labores de docencia en las instituciones educativas. También se indagó sobre el horario y la modalidad de formación que se ofrece en el programa; el $66 \%$ consideró favorable este aspecto. Si bien se planteó en uno de los apartes de este artículo que la proliferación y diversidad de programas de formación docente se caracterizan por la variedad de los procesos en que son desarrollados. Al respecto se cuestionó sobre el costo económico del programa, encontrándose que el $59 \%$ sintió interés por ese aspecto. Por otra parte, el $67 \%$ de los estudiantes está de acuerdo con que la calidad de la formación que se recibe en el programa es favorable. Por último, se encontró que el $52 \%$ de los entrevistados que decidieron cursar el programa lo han hecho por recomendación de un graduado (ver Tabla 2).

Tabla 2: Variables relacionadas con la motivación extrínseca

\begin{tabular}{|l|l|l|}
\hline $\begin{array}{l}\text { Variables relacionadas con } \\
\text { la motivación extrínseca }\end{array}$ & \multicolumn{1}{|c|}{ Contenido de la variable } & $\begin{array}{l}\text { Coefi- } \\
\text { cientes }\end{array}$ \\
\hline 8.1. Ejercer la docencia & $\begin{array}{l}\text { Requisito para ejercer labores en docencia en instituciones educativas } \\
\text { publicas/ privadas. }\end{array}$ & 0.873 \\
\hline $\begin{array}{l}\text { 8.3. Prácticas de } \\
\text { enseñanza }\end{array}$ & Mejorar las prácticas de enseñanza. & 0.890 \\
\hline 8.4. Sueldo & Mejorar ingresos económicos. & 0.862 \\
\hline 8.7. Vinculación adicional & $\begin{array}{l}\text { Porque puedo tener la oportunidad de contar con otra vinculación adicional } \\
\text { en instituciones educativas. }\end{array}$ & 0.858 \\
\hline $\begin{array}{l}\text { 8.8 horario y modalidad de } \\
\text { formación }\end{array}$ & Por el horario y modalidad de formación que se ofrece en el programa. & 0.851 \\
\hline 8.9 valor económico & Por el valor económico del programa. & 0.848 \\
\hline $\begin{array}{l}\text { 8.10 Calidad de la } \\
\text { formación }\end{array}$ & La calidad de la formación que se recibe en el programa. & 0.863 \\
\hline $\begin{array}{l}\text { 8.11 Recomendación del } \\
\text { programa }\end{array}$ & Por recomendación de un graduado del programa & 0.862 \\
\hline
\end{tabular}

\section{Factor 3. Percepción sobre el aporte del PPPNL en la formación y cualificación del perfil docente}

El $95 \%$ de los participantes afirmó que cursaba el PPPNL porque era un requisito para ejercer labores de docencia en las instituciones educativas. Al respecto, Marcelo y Vaillant (2015) afirman que "ser docente en el siglo XXI debe suponer para los miembros de la profesión asumir que el conocimiento y los estudiantes (...) cambian (...). Y que para dar respuesta adecuada y satisfacer el derecho de aprender de los estudiantes, se impone hacer un esfuerzo redoblado por seguir aprendiendo" (p. 23). El 93 \% de los estudiantes, al iniciar el programa, tiene como expectativa adquirir conocimientos en pedagogía; sin embargo, cuando se les indaga después de transcurrido el desarrollo de los diferentes seminarios, solo el $52 \%$ afirma que ha apropiado un amplio conocimiento en pedagogía, mientras 
que el $48 \%$ restante percibió que el programa no cumplió con las expectativas esperadas. Este resultado converge con los hallazgos de Castro y Barrantes (2011), en cuanto a que los PPPNL se quedan escasos en la formación en pedagogía, como consecuencia de las políticas institucionales para su desarrollo; por lo tanto, se hace necesario fortalecer el programa de tal manera que responda a la formación en pedagogía que requieren los estudiantes. El ejercicio de la profesión docente requiere del saber de la pedagogía, por tal razón es indispensable que el proceso de formación del educador no licenciado los prepare en esta dirección. Al indagar sobre la incidencia del espacio académico "Conceptualización y Fundamentos de Pedagogía"; el $46 \%$ de los participantes está de acuerdo en que el aporte de este seminario a su perfil de educador fue favorable, sin embargo, el $54 \%$ restante está en desacuerdo con tal afirmación. La evaluación comprendida como un proceso permanente e integral de seguimiento a los estudiantes, exige al educador la implementación de estrategias y mediaciones pedagógicas conducentes a optimizar la enseñanza y con ello el aprendizaje de los estudiantes. En esta línea, un planteamiento que sobresale desfavorablemente en el programa tiene que ver con la variable la evaluación aplicada a los estudiantes se toma como un proceso para cualificar el perfil del educador no licenciado; en relación con esta pregunta el 70 \% de los participantes manifestó estar en desacuerdo (ver Tabla 3).

Tabla 3: Variables relacionadas con la percepción sobre el aporte del PPPNL en la formación y cualificación del perfil docente

\begin{tabular}{|c|c|c|}
\hline $\begin{array}{c}\text { Variables relacionadas } \\
\text { con la percepción sobre } \\
\text { el aporte del PPPNL en } \\
\text { la formación y } \\
\text { cualificación del perfil } \\
\text { docente } \\
\end{array}$ & $\begin{array}{c}\text { Contenido de la variable } \\
\text { Procesos formativos en el Programa de Pedagogía para Profesionales no } \\
\text { Licenciados (PPPNL) }\end{array}$ & $\begin{array}{l}\frac{2}{2} \\
\frac{d}{0} \\
\frac{0}{0} \\
0 \\
0\end{array}$ \\
\hline $\begin{array}{l}\text { 9.13. metodología de los } \\
\text { docentes del programa }\end{array}$ & $\begin{array}{l}\text { Las metodologías de formación desarrolladas por los docentes del programa } \\
\text { promueven el dialogo deliberativo y reflexión constructiva sobre los conceptos de } \\
\text { Pedagogía y su relación con otras disciplinas presentes en la educación. }\end{array}$ & 0.932 \\
\hline $\begin{array}{l}9.14 \text { característica de las } \\
\text { clases }\end{array}$ & En los diferentes espacios académicos prevalecen las clases magistrales. & 0.932 \\
\hline 9.15 Evaluación & $\begin{array}{l}\text { La evaluación aplicada a los estudiantes se toma como un proceso para cualificar } \\
\text { el perfil del educador no licenciado. }\end{array}$ & 0.928 \\
\hline $\begin{array}{l}9.16 \text { nuevas tendencias } \\
\text { educativas }\end{array}$ & $\begin{array}{l}\text { Se hace evidente en la formación en pedagogía las nuevas tendencias educativas } \\
\text { en las que se desarrolla la actual educación colombiana. }\end{array}$ & 0.922 \\
\hline $\begin{array}{l}9.17 \text { apoyo } \\
\text { extracurricular }\end{array}$ & $\begin{array}{l}\text { Los estudiantes del programa reciben tutorías adicionales cuando presentan } \\
\text { dificultades académicas en algunos espacios académicos. }\end{array}$ & 0.933 \\
\hline $\begin{array}{l}9.18 \text { plan de trabajo } \\
\text { sincrónico }\end{array}$ & $\begin{array}{l}\text { El trabajo sincrónico (presencial) tenido en cuenta para los cursos contemplados } \\
\text { en el programa es suficiente para que los estudiantes apropien saberes amplios } \\
\text { en Pedagogía. }\end{array}$ & 0.927 \\
\hline $\begin{array}{l}9.19 \text { Calidad del } \\
\text { programa }\end{array}$ & La calidad del Programa de Pedagogía para Profesionales no Licenciados es alto. & 0.922 \\
\hline $\begin{array}{l}9.20 \text { Recomendación del } \\
\text { programa }\end{array}$ & $\begin{array}{l}\text { A partir de mi experiencia como estudiante del Programa lo recomendaría a una } \\
\text { persona. }\end{array}$ & 0.922 \\
\hline $\begin{array}{l}9.21 \text { Conocimiento } \\
\text { Transdisciplinar }\end{array}$ & $\begin{array}{l}\text { Se promueve el conocimiento transdisciplinar en los diferentes espacios } \\
\text { académicos del programa (se interrelacionan los contenidos de los cursos). }\end{array}$ & 0.924 \\
\hline $\begin{array}{l}9.22 \text { Conocimiento en } \\
\text { pedagogía }\end{array}$ & $\begin{array}{l}\text { El espacio académico "Conceptualización y fundamentos de la Pedagogía", } \\
\text { amplio mi conocimiento en Pedagogía de manera significativa. }\end{array}$ & 0.923 \\
\hline $\begin{array}{l}9.23 \text { incidencia en el } \\
\text { perfil de educador }\end{array}$ & $\begin{array}{l}\text { El espacio académico "Psicología educativa" incidió en mi perfil como educador } \\
\text { de manera significativa. }\end{array}$ & 0.922 \\
\hline $\begin{array}{l}9.24 \text { el uso de las TIC's } \\
\text { en la educación }\end{array}$ & $\begin{array}{l}\text { El espacio académico " Los Ambientes de Aprendizaje y las TIC" me mostraron } \\
\text { la importancia de involucrarlos en mi labor educativa. }\end{array}$ & 0.922 \\
\hline $\begin{array}{l}9.25 \text { Conocimiento de } \\
\text { herramientas de } \\
\text { investigación }\end{array}$ & $\begin{array}{l}\text { Considero que en el espacio académico " Investigación y Planeación de } \\
\text { proyectos" se brindaron las herramientas necesarias para llevarlas a la práctica } \\
\text { educativa. }\end{array}$ & 0.921 \\
\hline $\begin{array}{l}9.26 \text { Modelo de } \\
\text { formación }\end{array}$ & $\begin{array}{l}\text { El modelo de formación desarrollado por los docentes del Programa de } \\
\text { Pedagogía para Profesionales no Licenciados, se enmarca en el sustento teórico } \\
\text { del enfoque de la Praxeología Pedagógica que identifica a UNIMINUTO. }\end{array}$ & 0.922 \\
\hline $\begin{array}{l}9.27 \text { Aporte del espacio } \\
\text { académico }\end{array}$ & $\begin{array}{l}\text { El espacio académico de " Políticas Educativas" aporto de manera significativa } \\
\text { en mi formación como docente. }\end{array}$ & 0.921 \\
\hline $\begin{array}{l}9.28 \text { Conocimiento de } \\
\text { los modelos } \\
\text { pedagógicos }\end{array}$ & $\begin{array}{l}\text { El espacio académico " Modelos Pedagógicos" aportó significativamente a mi } \\
\text { conocimiento en el tema. }\end{array}$ & 0.923 \\
\hline $\begin{array}{l}9.29 \text { Conocimiento sobre } \\
\text { teorías del currículo }\end{array}$ & $\begin{array}{l}\text { El espacio académico " Teorías del Currículo" incidió significativamente en mi } \\
\text { formación en Pedagogía. }\end{array}$ & 0.923 \\
\hline $\begin{array}{l}\text { 9.30 Aplicación del } \\
\text { conocimiento }\end{array}$ & $\begin{array}{l}\text { Para la elaboración del Proyecto de Aula, requiere poner en práctica los } \\
\text { conocimientos apropiados en los diferentes espacios académicos del programa. }\end{array}$ & 0.922 \\
\hline
\end{tabular}


Una vez desagregados estos resultados, en función de generar un espacio de discusión que aporte a la implementación de posibles estrategias de resolución a las problemáticas identificadas, resulta pertinente confrontarlos con la opinión que algunos autores han expresado al respecto.

Estos resultados son los primeros indicios que identifican la necesidad de plantear acciones que contribuyan a la optimización del PPPNL y con ello a la calidad de la formación de los educadores no licenciados. En el orden señalado, de los hallazgos presentados se colige que es pertinente que al finalizar el programa se satisfaga un gran porcentaje de los intereses que manifiestan los estudiantes al iniciar sus estudios; por tanto, es preciso establecer un plan de seguimiento al proceso formativo. El programa estudiado está propuesto para ser desarrollado en cinco (5) meses aproximadamente, con una intensidad de 480 horas en total, para otorgar diez (10) créditos; dado su carácter semipresencial y autoformador, el criterio que se asume como más importante para evaluar el desempeño de los estudiantes es la participación activa en cada una de las sesiones. Sin embargo, como se indicó anteriormente, los resultados se muestran desfavorables en este aspecto. Otro detalle que se debe resaltar es la actualización del currículo del programa, puesto que no se ha tenido en cuenta que en cinco años las necesidades y los retos de formación docente han cambiado y aumentado. A partir de los resultados de esta investigación, se hace evidente que el programa tiene que trabajar en fortalecer la estructura curricular a fin de que la formación en pedagogía sea transversal en todos los espacios académicos.

Este señalamiento converge con los hallazgos y las conclusiones de García et al. (2014) cuando afirman que "modificar los requisitos del Registro Calificado para los programas de pedagogía, de manera que coincidan gradualmente con los estándares de Acreditación de Alta Calidad" (p. 31) es una prioridad para lograr la excelencia en los programas de formación docente. Los hallazgos que se detallaron en este estudio orientan a pensar en un programa en el que se tenga un avance de diez créditos que estén ligados a la obtención de un título de postgrado, bien sea a nivel de especialización o de maestría. Es posible que esta estrategia para ofrecer el programa dirigido a los profesionales no licenciados, con el fin de que den continuidad a sus estudios de postgrado, tenga mayores ventajas en la formación y la cualificación del perfil del educador que no cuenta con conocimientos en pedagogía y, con ello, se aporte al mejoramiento de la calidad de la educación colombiana. Otra de las estrategias de intervención que surgió después de la reflexión fue la propuesta de reestructuración de los seminarios del programa, de modo que se planeen de tal manera que tengan a la pedagogía como propósito fundante, no solo desde la teoría sino desde su aplicación en los contextos educativos. En relación con la incidencia del programa en el perfil del educador, el $43 \%$ de los participantes percibió estar de acuerdo con que apropiaron un conocimiento amplio en pedagogía; el 57 \% restante dijo estar en desacuerdo. En este sentido, es importante generar espacios de encuentro con docentes y estudiantes 0 , en su defecto, con representantes, en los que se delibere acerca de las dificultades y posibles acciones de mejora que permitan superar las situaciones que han incidido desfavorablemente en la formación de los profesionales no licenciados.

En la figura 1 se puede observar que el $92 \%$ de los estudiantes desarrollan labores de docencia sin haber cursado el Programa de Pedagogía para Profesionales No Licenciados, solo el $8 \%$ de los indagados manifiesta no tener experiencia en docencia, situación que no favorece los procesos de calidad de la educación colombiana si se considera que la competencia profesional docente está condicionada al saber pedagógico. La figura 1 muestra que en Colombia, para ejercer la docencia, solo se requiere superar un concurso en el que el saber específico de una disciplina se sobrepone al saber pedagógico que identifica la profesión docente. En este sentido, se evidencia la necesidad de que los PPPNL, en cuanto a pedagogía y didácticas, tengan una mayor cercanía a la formación que recibe un licenciado en educación.

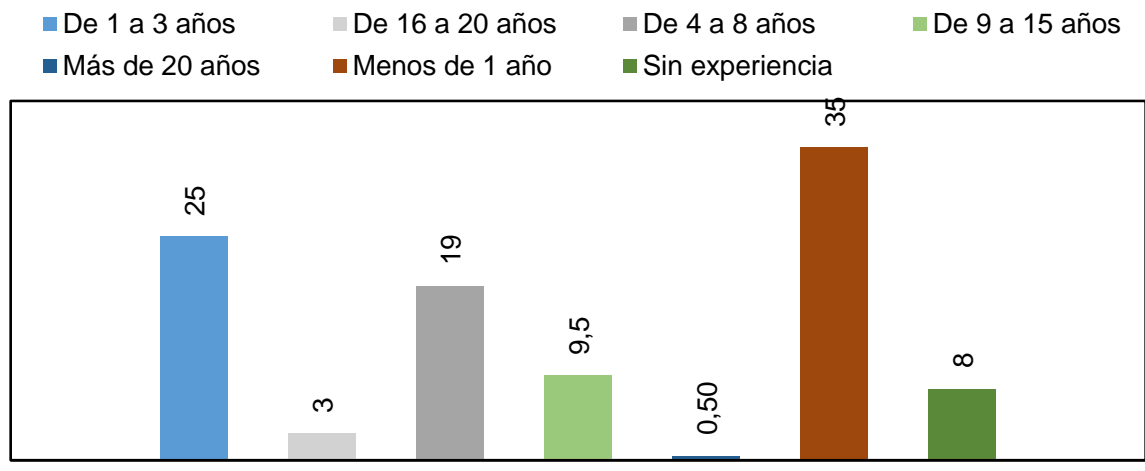

GRUPOS DE EXPERIENCIA \%

Fig. 1. Experiencia docente antes de iniciar el PPPNL. 
Si bien se expuso en este artículo que para ejercer la docencia se hace necesario contar con un amplio conocimiento en pedagogía y demás disciplinas asociadas a ella, los resultados muestran que los profesionales no licenciados desarrollan la labor docente sin contar previamente con un saber en educación; es decir, los planteamientos dados en la política colombiana en torno a la formación de los maestros difieren con las realidades de su vinculación al magisterio.

\section{CONCLUSIONES}

De acuerdo con los resultados de este estudio, y de su discusión y análisis, se plantean a continuación cuatro conclusiones: 1) a todas luces, la profundización y la ampliación en tiempo de los seminarios en los que se aborda la pedagogía es necesaria y conveniente para la formación y cualificación del perfil del educador no licenciado; 2) los diez créditos que se otorgan en el PPPNL, al ser homologados en estudios de postgrado (especialización o maestría), optimizan la formación en pedagogía de los estudiantes; 3) la actualización del programa, con base en las demandas de formación en pedagogía del profesional no licenciado, contribuye en la cualificación del perfil del educador y; 4) cuando presentan dificultades académicas, las tutorías extracurriculares para estudiantes son una herramienta que favorece la formación del educador no licenciado durante el desarrollo del programa.

\section{REFERENCIAS}

Acosta, L. A., O. Abreu y M. F. Coronel, Sistema de Formación Pedagógica en la Universidad de Otavalo en Ecuador, CIT, Formación Universitaria, 8(2), 43-52 (2015)

Aguerrondo, I., Hacia una Política Integral para la Formación y el Desarrollo Profesional de los Maestros de Educación Básica, 1ª Ed., pp. 25-29. Secretaria de Educación Pública, México, D. F., México (2003)

Aguerrondo, I., Formación Docente en la Sociedad del Conocimiento, Estudos em Avaliação Educacional, 20(44), 363-387 (2009)

Ariza, R.N., Formación de Docentes, El Gran Reto de la Calidad (en la web: https://goo.gl/fHEa6D, acceso: 27 de diciembre de 2016), Ministerio de Educación Nacional, Bogotá, D.C., Colombia (2014)

Castro, V.J., y R. Barrantes, Hacia una Caracterización de los Profesionales No Licenciados en Educación (PNL), Percepciones y Realidades, $1^{\text {a } E d ., ~ p p . ~ 31-84 . ~ A s c o f a d e, ~ B o g o t a ́, ~ D . ~ C ., ~ C o l o m b i a ~(2011) ~}$

Corporación Universitaria Minuto de Dios - UNIMINUTO, Programa Curricular de Pedagogía para Profesionales No Licenciados, PPPNL. Bogotá, D. C., Colombia (2011)

Cuervo, L.C., La Inserción de profesionales Docentes No Licenciados al Sistema Público Educativo, ¿Qué Hay en su Quehacer Pedagógico?, Grupo de Investigación "Paz desde la Paz", Facultad de Educación, Universidad Pedagógica y Tecnológica de Colombia, Bogotá, D.C., Colombia (2013)

Day, C., Pasión por enseñar, la identidad personal y profesional del docente y sus valores, 15-17, Narcea, Madrid, España (2006)

Decreto 1278 de 2002: “Estatuto de Profesionalización Docente”, Diario Oficial 44.840, Bogotá, D.C., Colombia (2002)

Decreto 2035 de 2005: "Objetivos y Requisitos del Programa de Pedagogía para Profesionales No Licenciados en Educación”, Diario Oficial 45.942, Bogotá, D.C., Colombia (2005)

Departamento Nacional de Planeación - DNP, Plan Nacional de Desarrollo 2014-2018, Tomo 1 (en la web: https://goo.gl/KNvk84, acceso: 29 de abril de 2017), Bogotá, D. C., Colombia (2015)

Durán, S. F., Z. D. Acosta y M. O. Espinel, Experiencia Docente de Profesionales No Licenciados en la Escuela Pública del Distrito Capital, Actualidades Pedagógicas, (63), 39-60 (2014)

García, S., D. Maldonado, G. Perry, C. Rodríguez y J. E. Saavedra, Tras la Excelencia Docente: Cómo Mejorar la Calidad de la Educación para todos los Colombianos, $1^{a}$ Ed., pp. 30-41, Fundación Compartir, Bogotá, D.C. Colombia (2014) 
Giroux. H., La Educación Superior y las Políticas de Ruptura. Disponible en: https://goo.gl/qtvwzT. Revista Entramados: educación y sociedad, Año 3, №3, 15-26 (2016)

Giroux, H., Los Profesores como Intelectuales: Hacia una Pedagogía Crítica del Aprendizaje, reimpresión de la 7ª Ed., Paidós-lbérica, Barcelona, España (1997)

Hernández, S.R., C.C. Fernández y L. P. Baptista, Metodología de la Investigación, $5^{a}$ Ed., 52-72, McGrawHill, Lima, Perú (2010)

Herrera, L., A.M. Fernández, K. Caballero y J.M. Trujillo, Teaching Competencies of Beginning Teachers participating in a Mentorship Project, Implications for University Professional Development, Profesorado: Revista de Currículum y Formación del Profesorado, 15(3), 217 (2011)

Herrera, T.L., B.A. Fernández., R. K. Caballero y T. J. Trujillo, Teaching Competencies of Beginning Teachers Participating in a Mentorship Project. Implications for University Professional Development, Profesorado: Revista de Currículum y Formación Del Profesorado, 15(3), 213-241 (2011)

Imbernón, M. F., La Formación Inicial y la Formación Permanente del Profesorado, Dos Etapas de un Mismo Proceso, Revista Interuniversitaria de Formación del Profesorado, (6), 487-499 (1989)

Juliao, V.C., Una Pedagogía Praxeológica, 1ª Ed., pp. 17-55. UNIMINUTO, Bogotá, D. C., Colombia (2014)

Ley 115 de 1994, "Ley General de Educación”, Artículo 72ํㅗan Nacional de Desarrollo Educativo, Diario Oficial 41.214, Bogotá, D. C., Colombia (1994)

Marcelo, C. y D. Vaillant, Desarrollo Profesional Docente, ¿Cómo se Aprende a Enseñar?, 1a Ed., pp. 22-49, Narcea, Madrid, España (2009)

Ministerio de Educación Nacional - MEN, Plan Nacional Decenal de Educación (PNDE) 2006-2016, 40-60, MEN, Bogotá, D.C., Colombia (2006)

Ministerio de Educación Nacional - MEN, Plan Sectorial 2010-2014, Documento 14, 24-26. Bogotá, D. C., Colombia (2010)

Rentería, R.P., Formación de Docentes, Un Reto para las Escuelas Normales Superiores y las Facultades de Educación, 1ª Ed., pp., 66-69, Cooperativa Editorial Magisterio, Bogotá, D. C., Colombia (2004)

Vaillant, D., SOS Profesión Docente: Al Rescate del Curriculum Escolar, IBE Working Papers on Curriculum, 2, 9-16, Unesco, Ginebra, Suiza (2006)

Vaillant, D., Iniciativas Mundiales para Mejorar la Formación de Profesores, Revista Brasilera de Estudios Pedagógicos, 91(229), 543-561 (2010)

Vaillant, D. y C. Marcelo, El ABC y D de la Formación Docente, 134, 52-53, Narcea, Madrid, España (2015)

Vezub, L. F., La formación y el Desarrollo Profesional Docente Frente a los Nuevos Desafíos de la Escolaridad, Curriculum y Formación del Profesorado, 11(1), 16-18 (2007) 
